\title{
Optimal Residential Load Control with Price Prediction in Real-Time Electricity Pricing Environments
}

\author{
Amir-Hamed Mohsenian-Rad, Member, IEEE and Alberto Leon-Garcia, Fellow, IEEE
}

\begin{abstract}
Real-time electricity pricing models can potentially lead to economic and environmental advantages compared to the current common flat rates. In particular, they can provide end users with the opportunity to reduce their electricity expenditures by responding to pricing that varies with different times of the day. However, recent studies have revealed that the lack of knowledge among users about how to respond to timevarying prices as well as the lack of effective building automation systems are two major barriers for fully utilizing the potential benefits of real-time pricing tariffs. We tackle these problems by proposing an optimal and automatic residential energy consumption scheduling framework which attempts to achieve a desired trade-off between minimizing the electricity payment and minimizing the waiting time for the operation of each appliance in household in presence of a real-time pricing tariff combined with inclining block rates. Our design requires minimum effort from the users and is based on simple linear programming computations. Moreover, we argue that any residential load control strategy in real-time electricity pricing environments requires price prediction capabilities. This is particularly true if the utility companies provide price information only one or two hours ahead of time. By applying a simple and efficient weighted average price prediction filter to the actual hourly-based price values used by the Illinois Power Company from January 2007 to December 2009, we obtain the optimal choices of the coefficients for each day of the week to be used by the price predictor filter. Simulation results show that the combination of the proposed energy consumption scheduling design and the price predictor filter leads to significant reduction not only in users' payments but also in the resulting peak-to-average ratio in load demand for various load scenarios. Therefore, the deployment of the proposed optimal energy consumption scheduling schemes is beneficial for both end users and utility companies.
\end{abstract}

Keywords: Wholesale electricity market, real-time pricing, inclining block rates, price prediction, energy consumption scheduling.

\section{INTRODUCTION}

Since electricity is non-storable economically, wholesale prices (i.e., the prices set by competing generators to regional electricity retailers) vary from day to day and usually fluctuate by an order of magnitude between low-demand night-time hours to high-demand afternoons. However, in general, almost all retail consumers are currently charged some average price that does not reflect the actual wholesale price at the time of consumption [1]. As a remedy to this problem, various time-differentiated pricing models have been proposed: realtime pricing (RTP), day-ahead pricing (DAP), time-of-use

Manuscript was received on January 29, 2010; revised on May 17, 2010; and accepted on June 7, 2010.

The authors are with the Department of Electrical and Computer Engineering, University of Toronto, Toronto, ON, Canada, M5S 2E4, e-mails: hamed@comm.utoronto.ca and alberto.leongarcia@utoronto.ca. pricing (TOUP), critical-peak pricing (CPP), etc. In all of these variations, the main idea is two-fold: First, allowing retail prices to reflect fluctuating wholesale prices to the end users so that they pay what the electricity is worth at different times of the day; Second, encouraging users to shift high-load household appliances to off-peak hours to not only reduce their electricity costs but also to help to reduce the peak-to-average ratio (PAR) in load demand ${ }^{1}$ [2]-[5].

The research literature includes a wide range of work related to RTP. The earliest peak-load pricing discussion dates more than half a century ago [6], [7]. More recent theoretical and simulation studies in [8]-[12] have focused on understanding the economic advantages of RTP. Many of these (e.g., [10], [11]) have proposed carefully designed tariff models in order to improve system performance and users' participation in RTP and CPP programs. On the other hand, the environmental implications of RTP are examined in [13] and it is shown that RTP can potentially reduce the emission levels of $\mathrm{SO}_{2}$, $\mathrm{NO}_{\mathrm{x}}$, and $\mathrm{CO}_{2}$ in many regions in the U.S., where peak demand is met more by oil-fired capacity than by hydropower. Time-differentiated pricing is currently implemented in various regions in North America, e.g., in form of hourly-based DAP tariff used by the Illinois Power Company in the U.S. [14] and the three-level (on-peak, mid-peak, off-peak) TOUP tariff used by the Ontario Hydro Company in Toronto, Canada [15].

Another alternative to the common flat rates in retail electricity market is the conservation rates model with inclining block rates (IBR). In IBR pricing, the marginal price increases by the total quantity consumed [16]. That is, beyond a certain threshold in the total monthly/daily/hourly residential load, the electricity price will increase to a higher value. This creates incentives for end users to conserve to distribute their load at different times of the day in order to avoid paying for electricity at higher rates. In addition, IBR helps in load balancing and reducing the PAR [17]. It has been widely adopted in the pricing tariffs by some utility companies since the 1980s. For example, the Southern California Edison, San Diego Gas \& Electric, and Pacific Gas \& Electric companies currently have two-level residential rate structures where the marginal price in the second level (i.e., the higher block) is $80 \%$ or higher than the first level (i.e., the lower block), depending on the utility [18]. In Canada, the British Columbia

${ }^{1}$ Appropriate load-shifting is foreseen to become even more crucial as plugin hybrid electric vehicles (PHEVs) become more popular. Most PHEVs need $0.2-0.3 \mathrm{kWh}$ of charging power for one mile of driving [2]. This will represent a major new load on the existing distribution system. In particular, during the charging time, the PHEVs double the average household load [2]. 
Hydro Company currently uses a two-level conservation rate structure with $40 \%$ higher prices at the second level [19].

Recent studies have shown that despite several advantages that RTP and IBR can offer, the lack of knowledge among the users about how to respond to time-varying prices and the lack of effective home automation systems are two major barriers for fully utilizing the benefits of real-time pricing tariffs [20], [21]. In fact, most of the current residential load control activities are operated manually. This makes it difficult for users to optimally schedule the operation of their appliances in response to the hourly updated pricing information they may receive from the utilities in an RTP program. For example, the experience of the RTP program in Chicago has shown that although the price values were available via telephone and the Internet, only rarely did households actively check prices as it was difficult for the participants to constantly monitor the hourly prices to respond properly [1]. Another example is the results from a more recent study by The Utility Reform Network (TURN) in San Francisco which has reported that most users do not have time and knowledge to even pursue their own interest while they respond to real-time prices [22].

The main focus of this paper is on proposing a computationally feasible and automated optimization-based residential load control scheme in a retail electricity market with RTP combined with IBR. We aim to minimize the household's electricity payment by optimally scheduling the operation and energy consumption for each appliance, subject to the special needs indicated by the users. We assume that each residential consumer is equipped with a smart meter [23], connected to a smart power distribution system with a two-way digital communication capability through computer networking [24][26]. While periodically receiving the updated information on prices from the utility, each smart meter includes an energy scheduling unit which decides on energy consumption in the household. Depending on the scheduling horizon, the operation of the energy scheduling unit is complemented by a price predictor unit which estimates the upcoming prices by applying a weighted averaging filter to past prices. We obtain the optimal coefficients of the price predictor filter and show that it is best to use different coefficients at different days of the week. In this regard, we use the actual RTP tariffs adopted by the Illinois Power Company (IPC) from January 2007 to December 2009 which was available to public online at [27].

The results and analysis in this paper differ from the related work in the literature in several aspects. Unlike [9] we do not focus on understanding the residential user's response to RTP models. Instead, we try to help the users to shape their response properly and in an automated fashion. Our work is also different from the heuristic home automation schemes in [21], [28] as here we use an optimization-based approach with elaborate mathematical analysis. Furthermore, the IPbased networking architecture proposed for home automation in [21] can also be used for the implementation of our design in practice. While the optimization problem we study in this paper is partly similar to the one studied in [17] for fixed prices, here we take into account time-varying prices as well as the trade-off between minimizing the electricity payment and minimizing the waiting time for the operation of each appliance. In addition, the optimization problem we study here is more realistic with respect to price models but requires more efforts to be solved due to the non-differentiability of the objective function. Last but not least, price prediction is not studied in [9], [17]. In fact, to the best of our knowledge, none of the prior work on residential load control has considered real-time price prediction at the user side.

The rest of this paper is organized as follows. We introduce the system model and notation in Section II. In Section III, we discuss the price prediction problem and introduce our weighted average price prediction filter which is designed and evaluated on a weekly basis, using the actual hourly price values adopted by the IPC. Our proposed linear programming scheme for optimal load control is introduced in Section IV. Remarks, special cases, and extensions are highlighted in Section V. Simulation results are provided and discussed in Section VI. The paper is concluded in Section VII.

\section{System MODEL}

In this section, we provide a mathematical representation of the residential load control problem in RTP environments with IBR. We consider the general wholesale electricity market scenario shown in Fig. 1, where each retailer/utility serves a number of end users. The RTP information, reflecting the wholesale prices, are informed by the retailer to the users over a digital communication infrastructure, e.g., a local area network (LAN). In this scenario, our focus is to formulate the energy consumption scheduling problem in each household as an optimization problem that aims to achieve a trade-off between minimizing the electricity payment and minimizing the waiting time for the operation of each household appliance in response to the real-time prices announced by the retailer company. We will explain how the optimization problem in this section can be solved in practice later in Section IV.

\section{A. Residential Consumers}

Consider a residential unit that participates in a real-time pricing program. Let $\mathcal{A}$ denote the set of appliances in this unit which may include washer/dryer, refrigerator, plug-in hybrid vehicle, etc. For each appliance $a \in \mathcal{A}$, we define an energy consumption scheduling vector $\mathbf{x}_{a}$ as follows:

$$
\mathbf{x}_{a} \triangleq\left[x_{a}^{1}, \ldots, x_{a}^{H}\right]
$$

where $H \geq 1$ is the scheduling horizon that indicates the number of hours ahead which are taken into account for decision making in energy consumption scheduling. For example, $H=24$ or $H=48$. For each upcoming hour of the day $h \in \mathcal{H} \triangleq\{1, \ldots, H\}$, a real-valued scalar $x_{a}^{h} \geq 0$ denotes the corresponding one-hour energy consumption that is scheduled for appliance $a \in \mathcal{A}$. On the other hand, let $E_{a}$ denote the total energy needed for the operation of appliance $a \in \mathcal{A}$. For example, in case of a plug-in hybrid electric sedan, in total $E_{a}=16 \mathrm{kWh}$ is needed to charge the battery for a 40-miles driving range [2]. As another example, for a typical front-loading clothes washing machine with warm wash/rinse setting, we have $E_{a}=3.6 \mathrm{kWh}$ per load [29]. Next, assume that for each appliance $a \in \mathcal{A}$, the user indicates $\alpha_{a}, \beta_{a} \in \mathcal{H}$ 


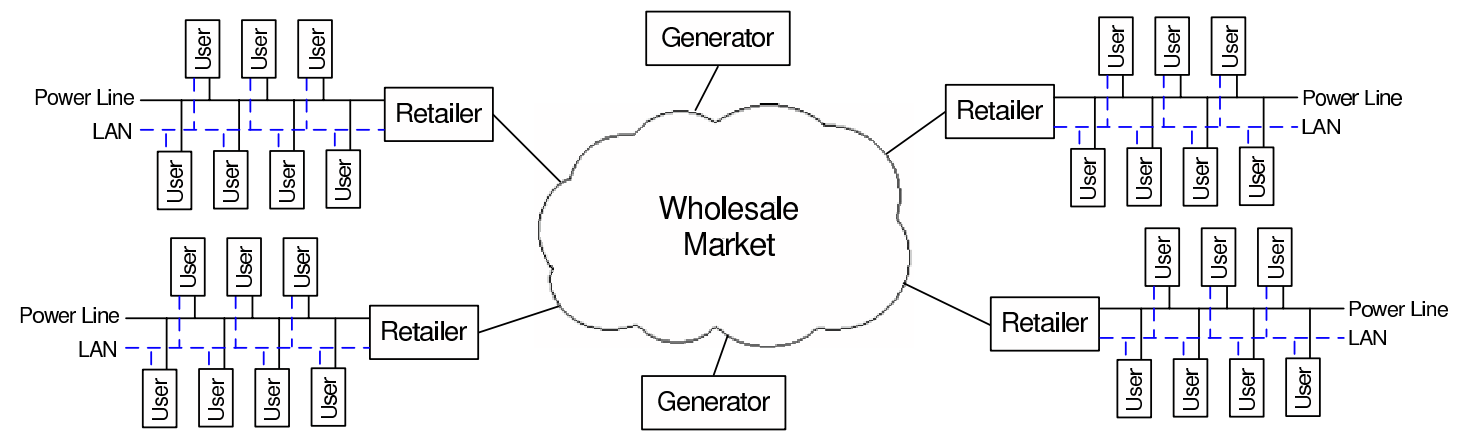

Fig. 1. A simplified illustration of the wholesale electricity market formed by multiple generators and several regional retail companies. Each retailer provides electricity for a number of users. Retailers are connected to the users via local area networks which are used to announce real-time prices to the users.

as the beginning and end of a time interval in which the energy consumption for appliance $a$ is valid to be scheduled, respectively. Clearly, we always have $\alpha_{a}<\beta_{a}$. For example, after loading a dishwasher with the dishes used at the lunch table, the user may select $\alpha_{a}=2 \mathrm{PM}$ and $\beta_{a}=6 \mathrm{PM}$ for scheduling the energy consumption for the dishwasher as he expects the dishes to be ready to use by dinner time in the evening. As another example, the user may select $\alpha_{a}=10 \mathrm{PM}$ and $\beta_{a}=7 \mathrm{AM}$ (the next day) for his PHEV after plugging it in at night such that the battery charging finishes by early morning time when he needs to use the vehicle to go to work. Given the pre-determined parameters $E_{a}, \alpha_{a}$, and $\beta_{a}$, in order to provide the needed energy for each appliance $a \in \mathcal{A}$ in times within the interval $\left[\alpha_{a}, \beta_{a}\right]$, it is required that

$$
\sum_{h=\alpha_{a}}^{\beta_{a}} x_{a}^{h}=E_{a} .
$$

Further to constraint (2), it is expected that $x_{a}=0$ for any $h<\alpha_{a}$ and $h>\beta_{a}$ as no operation (thus energy consumption) is needed outside the time frame $\left[\alpha_{a}, \beta_{a}\right]$ for appliance $a$. We note that the time length $\beta_{a}-\alpha_{a}$ needs to be larger than or equal to the time duration required to finish the normal operation of appliance $a$. For example, for a single-phase PHEV, the normal charging time is 3 hours [2]. Therefore, it is required that $\beta_{a}-\alpha_{a} \geq 3$. Clearly, if $\beta_{a}-\alpha_{a}=3$ the timing imposed by the user would be strict and any energy consumption scheduling strategy has no choice but arranging full power charging within the whole interval $\left[\alpha_{a}, \beta_{a}\right]$. On the other hand, if $\beta_{a}-\alpha_{a} \gg 3$, it is possible to select certain hours within the large interval $\left[\alpha_{a}, \beta_{a}\right]$ to schedule energy consumption such that the electricity payments can be minimized. We will further discuss this issue in Section II-C.

All home appliances have certain maximum power levels denoted by $\gamma_{a}^{\max }$, for each $a \in \mathcal{A}$. For example, a PHEV may be charged only up to $\gamma_{a}^{\max }=3.3 \mathrm{~kW}$ per hour [2]. Some appliances may also have minimum stand-by power levels $\gamma_{a}^{\text {min }}$, for each $a \in \mathcal{A}$. Therefore, the following lower and upper bound constraints are needed on the choices of the energy scheduling vector $\mathbf{x}_{a}$ for each appliance $a \in \mathcal{A}$ :

$$
\gamma_{a}^{\min } \leq x_{a}^{h} \leq \gamma_{a}^{\max }, \quad \forall h \in\left[\alpha_{a}, \beta_{a}\right] .
$$

Finally, we note that there is usually a limit on the total energy consumption at each residential unit at each hour. This limit, denoted by $E^{\max }$, can be set by the utility to impose the following set of constraints on energy scheduling:

$$
\sum_{a \in \mathcal{A}} x_{a}^{h} \leq E^{\max }, \quad \forall h \in \mathcal{H} .
$$

Together, constraints (2)-(4) determine all valid choices for the energy consumption scheduling vectors. Therefore, we can define a feasible scheduling set $\mathcal{X}$ for all possible energy consumption scheduling vectors as

$$
\begin{aligned}
& \mathcal{X}=\left\{\mathbf{x} \mid \sum_{h=\alpha_{a}}^{\beta_{a}} x_{a}^{h}=E_{a}, \forall a \in \mathcal{A},\right. \\
& \gamma_{a}^{\min } \leq x_{a}^{h} \leq \gamma_{a}^{\max }, \forall a \in \mathcal{A}, h \in\left[\alpha_{a}, \beta_{a}\right], \\
& x_{a}^{h}=0, \quad \forall a \in \mathcal{A}, h \in \mathcal{H} \backslash\left[\alpha_{a}, \beta_{a}\right] \text {, } \\
& \left.\sum_{a \in \mathcal{A}} x_{a}^{h} \leq E^{\max }, \quad \forall h \in \mathcal{H}\right\},
\end{aligned}
$$

where $\mathbf{x} \triangleq\left(\mathbf{x}_{a}, \forall a \in \mathcal{A}\right)$ denotes the vector of energy consumption scheduling variables for all appliances. An energy schedule $\mathbf{x}$ is valid only if $\mathbf{x} \in \mathcal{X}$. Clearly, the proper choice of $\mathbf{x}$ would depend on the electricity prices. In this regard, we assume that each household is equipped with a smart meter as shown in Fig. 2. The real-time prices are provided by the utility company via a LAN. The user announces his needs by selecting parameters $E_{a}, \alpha_{a}, \beta_{a}, \gamma_{a}^{\min }$, and $\gamma_{a}^{\max }$ for each appliance $a \in \mathcal{A}$. Then, the energy scheduler, with some help form the price predictor if needed, determines the optimal choice of energy consumption scheduling vector $\mathbf{x}$. The resulting energy consumption schedule is then applied to all household appliances in form of on/off commands with specified power levels over a wired or wireless home area network among the appliances and the smart meter. An example wireless home area network (WHAN) is shown in Fig. 3. In this setting, the in-home wireless communications can be implemented by ZigBee transceivers, offered by the ZigBee Alliance [30]. Another candidate for in-home communication is HomePlug power-line communication technology, offered by HomePlug Powerline Alliance [31]. More details about various home area network technologies can be found in [32].

Next, we discuss the details on the real-time pricing model as well as our proposed optimization-based load control strategy in Sections II-B and II-C, respectively. 


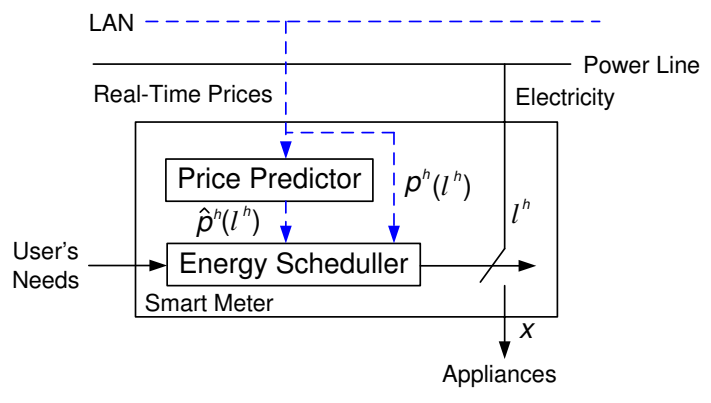

Fig. 2. The operation of smart meter in our design. Given the real-time prices $p^{h}\left(l^{h}\right)$ for all $h \in \mathcal{P}$ from the utility, there are two main units involved in residential load control: energy scheduler and price predictor. The latter estimates the upcoming prices which are not announced by the utility, i.e., $\hat{p}^{h}\left(l^{h}\right)$ for all $h \in \mathcal{H} \backslash \mathcal{P}$ if the price announcement horizon $P$ is less that the scheduling horizon $H$. The proper choices of energy consumption scheduling vectors $\mathbf{x}_{a}$ for all appliances $a \in \mathcal{A}$ is determined by the energy scheduler unit based on the solution of optimization problem (25).

\section{B. Real-Time Pricing with Inclining Block Rates}

Recall from Section I that RTP and IBR are two promising non-flat pricing models to replace the current flat rate tariffs. In this section, we provide a general mathematical pricing representation which combines these two pricing models. For now, we assume that the future pricing parameters are known for the users ahead of time. This is indeed the case in DAP structures. We will discuss price prediction in Section III.

Let $l^{h} \triangleq \sum_{a \in \mathcal{A}} x_{a}^{h}$ denote the total hourly household energy consumption at each upcoming hour $h \in \mathcal{H}$. Recall that $H$ denotes the scheduling horizon. We consider a general hourly pricing function $p^{h}\left(l^{h}\right)$ which depends on three parameters $a^{h}, b^{h}, c^{h} \geq 0$ and is formulated as follows:

$$
p^{h}\left(l^{h}\right)= \begin{cases}a^{h}, & \text { if } 0 \leq l^{h} \leq c^{h}, \\ b^{h}, & \text { if } l^{h}>c^{h} .\end{cases}
$$

It is clear that the price model in (6) is not a flat rate structure as the price value depends on time of day and total load. In fact, the price model in (6) represents an RTP structure combined with IBR. To see this, let us consider the example pricing models shown in Figs. 1(a) and (b) which are currently implemented by IPC in the U.S. and British Columbia Hydro Company in Canada, respectively. These examples are both special cases of the more general pricing model in (6). For the RTP model used by IPC in Fig. 1(a), we have

$$
a^{h}=b^{h}, \quad \forall h \in \mathcal{H} .
$$

That is, although the prices vary every hour, they are indeed flat within each hour. On the other hand, for the IBR used by British Columbia Hydro Company, we have

$$
\begin{aligned}
& a^{1}=a^{2}=\ldots=a^{H-1}=a^{H}, \\
& b^{1}=b^{2}=\ldots=b^{H-1}=b^{H}, \\
& c^{1}=c^{2}=\ldots=c^{H-1}=c^{H} .
\end{aligned}
$$

That is, although the prices are dependent on consumption level, they do not change over time; thus, they cannot reflect the fluctuations in the wholesale prices. By combining the two pricing scenarios in (6), both wholesale prices as well as consumption levels are taken into account.

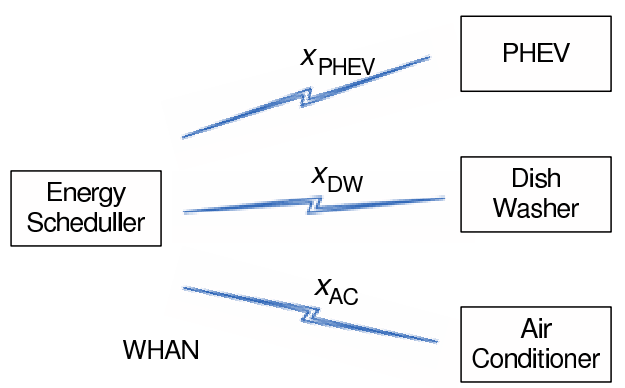

Fig. 3. The resulting energy consumption schedules selected by the energy scheduler can be applied to household appliances in form of operation commands over a wireless home area network using ZigBee transceivers.

\section{Problem Formulation}

Given the feasible energy scheduling set $\mathcal{X}$ and the RTP model in (6), the key question is: How should each user's energy consumption be scheduled in response to time-varying prices? Before answering this question, we first argue that the user's interest is two fold. First, each user wishes to minimize his payment. In fact, it is reasonable to assume that all users care about the amount on their electricity bills. Second, depending on the appliance, some users may also care about their comfort and getting the work done (e.g., washing their dishes, charging their PHEV, or cleaning their clothes) as soon as possible. Clearly, these two objectives can be conflicting in many scenarios. For example, in case of the RTP structure in Fig. 4(a) and when the user wants to start washing dishes at 9:00 AM right after finishing the breakfast, he may choose to wait for 5 hours and postpone the operation of the dishwasher (with $E_{a}=3.6 \mathrm{kWh}$ per load) to 2:00 PM in order to reduce the corresponding electricity payment from $3.6 \times 4.1=14.8$ cents to $3.6 \times 2.9=10.6$ cents and save 4.2 cents. However, for some reason, the user may prefer to pay the extra 4.2 cents and finish the work by 10:00 AM. As an alternative, the user might be willing to wait for 2 hours only and save 1.5 cents instead. In fact, we can see that there is a trade-off involved between the two design objectives. Next, we explain how this trade-off can be mathematically taken into account in an optimization-based framework.

From the RTP model introduced in Section II-B, the user's total electricity payment corresponding to all appliances within the upcoming scheduling horizon is obtained as

$$
\sum_{h=1}^{H} p^{h}\left(\sum_{a \in \mathcal{A}} x_{a}^{h}\right) \times\left(\sum_{a \in \mathcal{A}} x_{a}^{h}\right),
$$

where the price function $p^{h}(\cdot)$ is as in (6). On the other hand, the cost of waiting can be modeled as

$$
\sum_{h=1}^{H} \sum_{a \in \mathcal{A}} \rho_{a}^{h} x_{a}^{h}
$$

Here, for each appliance $a \in \mathcal{A}$ and any hour $h \in \mathcal{H}$, the waiting parameter $\rho_{a}^{h} \geq 0$. Clearly, $\rho_{a}^{h}=0$ for all $h<\alpha_{a}$ and $h>\beta_{a}$ as the concept of waiting may only be defined within the valid scheduling interval $\left[\alpha_{a}, \beta_{a}\right]$. On the other hand, it is reasonable to assume that we always have

$$
\rho_{a}^{\alpha_{a}} \leq \ldots \leq \rho_{a}^{\beta_{a}}, \quad \forall a \in \mathcal{A} .
$$




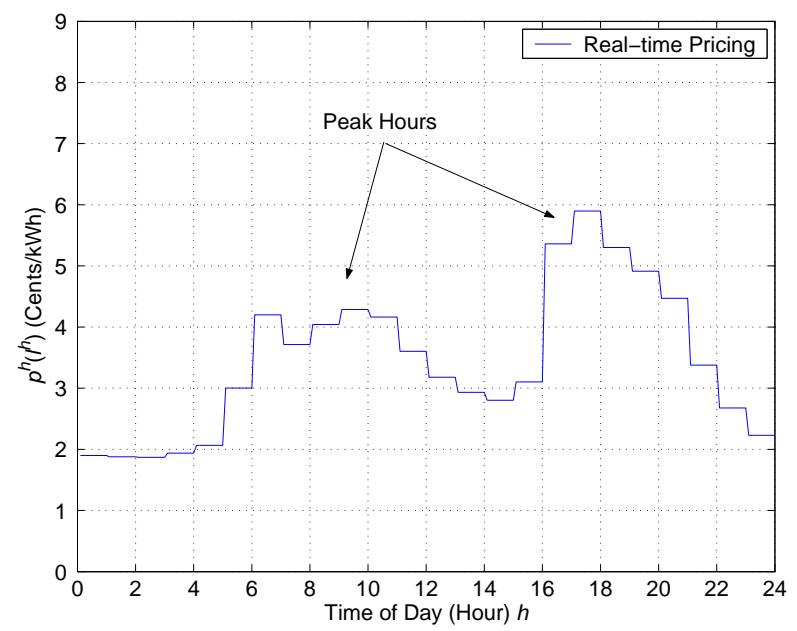

(a) Real-time prices set by Illinois Power Company.

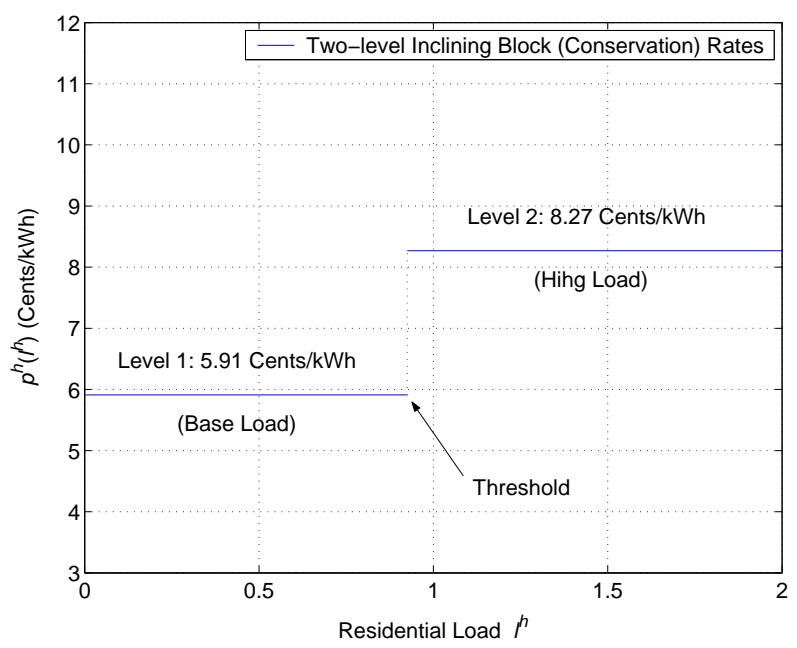

(b) Two-level inclining block rates set by BC Hydro.

Fig. 4. Examples of two non-flat pricing models. The real-time prices are used by Illinois Power Company on December 15, 2009 [14]. The inclining block rates are used by British Columbia Hydro in December 2009 [19].

That is, the cost of waiting increases as more energy consumption is scheduled at later hours. In particular, one can use the following model for the waiting parameter for each $a \in \mathcal{A}$ :

$$
\rho_{a}^{h}=\frac{\left(\delta_{a}\right)^{\beta_{a}-h}}{E_{a}}, \quad \forall a \in \mathcal{A}, h \in\left[\alpha_{a}, \beta_{a}\right],
$$

where $\delta_{a} \geq 1$ is an adjustable control parameter. The higher the value of parameter $\delta_{a}$ the higher will be the cost of waiting.

We are now ready to formulate the energy consumption scheduling problem as the following optimization problem:

$$
\begin{aligned}
\underset{x \in \mathcal{X}}{\operatorname{minimize}} & \sum_{h=1}^{H} p^{h}\left(\sum_{a \in \mathcal{A}} x_{a}^{h}\right)\left(\sum_{a \in \mathcal{A}} x_{a}^{h}\right) \\
& +\lambda_{\text {wait }} \sum_{h=1}^{H} \sum_{a \in \mathcal{A}} \frac{\left(\delta_{a}\right)^{\beta_{a}-h} x_{a}^{h}}{E_{a}},
\end{aligned}
$$

where the optimization variables are the energy consumption scheduling vectors $\mathbf{x}_{a}$ for all appliances $a \in \mathcal{A}$. The first and the second terms in the objective function in (15) denote the total electricity payment amount and the total cost of waiting across all appliances, respectively. Here, parameter $\lambda_{\text {wait }}$ is used in order to control the importance of the waiting cost terms in the objective function of the proposed design optimization problem. A typical value for this parameter can be $\lambda_{\text {wait }}=1$. On the other hand, parameter $\delta_{a}$ acts as a knob to control the trade-off between the two design objectives with respect to minimizing the payment and the waiting cost for each appliance. Clearly, a user may assign different values $\delta_{a}$ for different appliances. As a special case, we notice that if for a certain appliance $a \in \mathcal{A}$ we have $\delta_{a}=1$, then

$$
\sum_{h=1}^{H} \frac{\left(\delta_{a}\right)^{\beta_{a}-h} x_{a}^{h}}{E_{a}}=1, \quad \forall \mathbf{x} \in \mathcal{X},
$$

where the equality is due to (2). Therefore, the waiting cost will have no impact on the solution of optimization problem (15). In practice, three choices of parameter $\delta_{a}$ can be predetermined and labeled as three operation modes:

- Strict Cost Reduction: $\delta_{a}=1$,

- Medium Cost Reduction: $\delta>1$,

- No Cost Reduction: $\delta_{a} \gg 1$.

Next, we address the issue of price prediction. Then, we will explain how to solve problem (15) in practice with low computational complexity in Section IV. Note that optimization problem (15) is not tractable in its current form due to the non-differentiability of the price function $p^{h}\left(l^{h}\right)$ in (6).

\section{PRICE PREDICTION IN REAL-TIME PRICING ELECTRICITY ENVIRONMENTS}

So far, we have assumed that each end user is fully aware of the upcoming price values set by the utility company within the scheduling horizon $H$. That is, the user always knows the values of $a^{h}, b^{h}$, and $c^{h}$ for each $h \in \mathcal{H}$. This assumption can be valid in certain practical scenarios such as in DAP where the utility company releases the pricing details for the next 24 hours on a daily basis. Examples of such real-time pricing tariffs include the one implemented by IPC [14]. However, we may consider more dynamic pricing scenarios where the upcoming prices are announced only for $1 \leq P \ll H$ hours ahead of time. Here, $P$ denotes the price announcement horizon. For example, we may have $P=5$ or 6 hours. Clearly, the extreme case would be $P=1$ when only the next hour price is released. In these cases, any energy consumption scheduling policy, including the optimization-based energy consumption scheduling approach described in Section II-C, essentially requires some price prediction capabilities.

\section{A. Prediction Based on Prior Knowledge}

In general, price parameters may depend on several factors. In particular, they depend on the wholesale market prices which are not easy to predict themselves. Nevertheless, it is usually expected that the prices are higher during the afternoon, on hot days in the summer, and on cold days in the winter [1]. Furthermore, one may expect that the prices vary depending on the working days or weekends. These pieces of information can potentially help in predicting the 


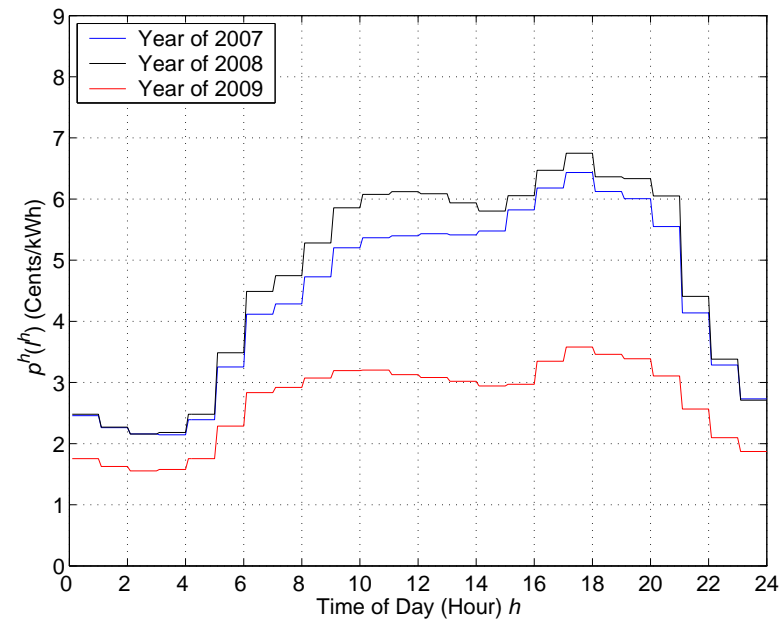

(a) Different Years

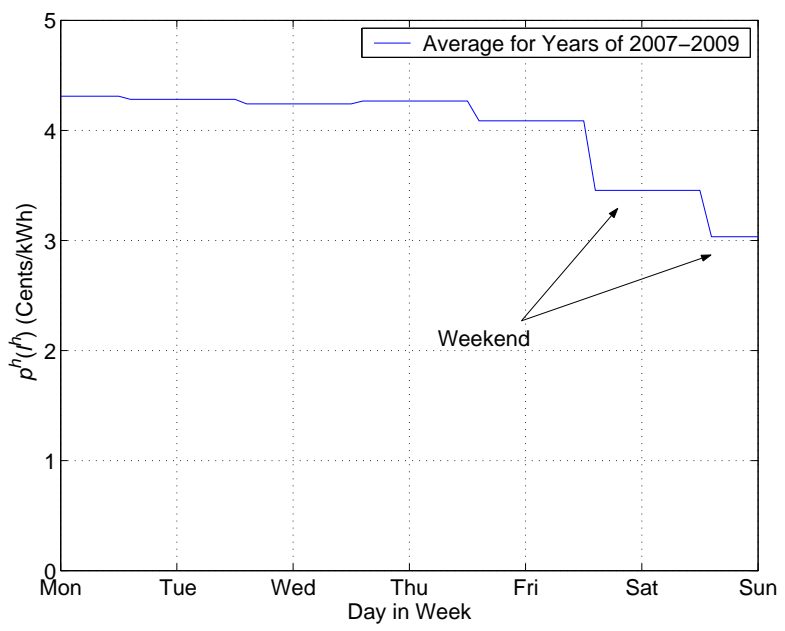

(c) Different Days of Week

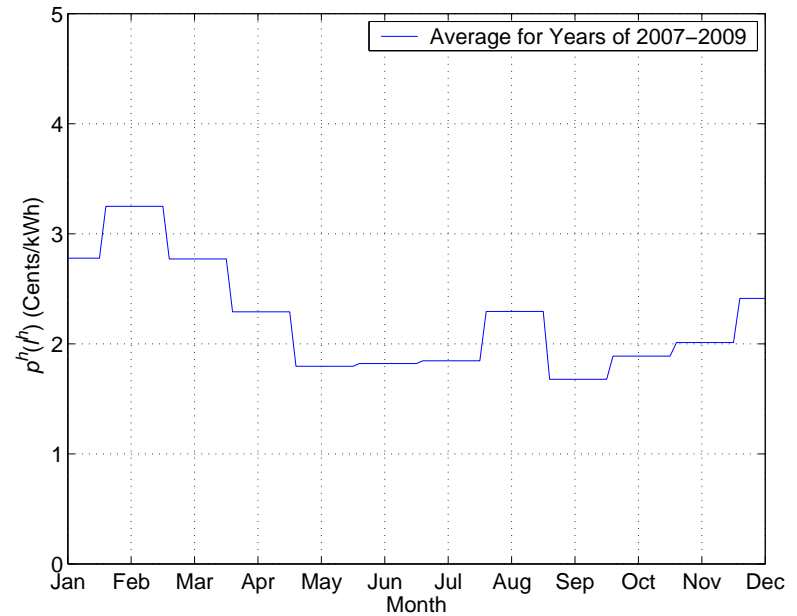

(b) Different Months of Year

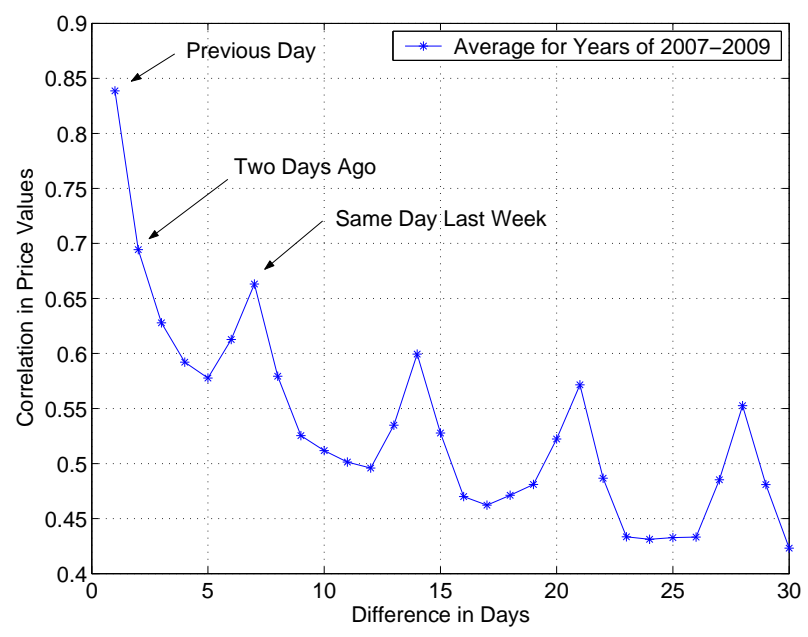

(d) Correlation with Past Prices at Different Days

Fig. 5. Statistical analysis of the real-time prices used by Illinois Power Company from January 2007 to December 2009.

price values in an RTP environment. While we are interested in accurate predictions, our main focus is to develop price predictors that have low computational complexity and can be implemented easily in residential smart meter along with the energy scheduling unit.

Next, we use the prices adopted by IPC from January 2007 to December 2009 to evaluate different factors that may affect RTP. The results are shown in Fig. 5. First, we plot the average hourly prices across all days of the year for the years of 2007 , 2008, and 2009 in Fig. 5(a). From the results in this figure, we can see that although the trends are partially (not exactly) similar in different years, the exact prices can be drastically different. This can be due to major yearly fluctuations in the wholesale prices, e.g., due to changes in the international price of oil. Therefore, making an accurate prediction based on the price values in the previous years does not seem feasible. Next, consider the average monthly prices (across all three years) in Fig. 5(b). We can see that there are significant differences in prices at different months. However, unlike what is usually expected (e.g., see [1]), the average prices are not always higher in the summer. Thus, it is not clear if we can predict prices based on the month of the year. Third, for the results in Fig. 5(c), we have plotted the average prices across all three years based on the day of the week. We can see that there are not major differences in average prices from Monday to Friday, except for slight price reduction on Friday. However, the prices on Saturday and Sunday are much less. This suggests that there can be relationships between the upcoming prices and whether the prices are for a working day or for a weekend. This is also shown in Fig. 5(d), where we plotted the correlation among the hourly-prices today with those in the previous days. From the results in this figure, we can see that there is a very high correlation (about 0.84 ) between the prices today and those yesterday. The correlation decreases as we go further back. However, there is also a noticeable correlation (about 0.67 ) between the prices today and those on the same day last week.

In summary, the observations in Fig. 5 suggest that an efficient prediction is likely by looking at the prices on yesterday, the day before yesterday, and the same day last week. Let 
TABLE I

Optimal Daily Coefficients for Price Predictor Filter

\begin{tabular}{|l||c|c|c|}
\hline Day & $k_{1}$ & $k_{2}$ & $k_{7}$ \\
\hline Monday & 0.355 & 0.465 & 0.359 \\
\hline Tuesday & 0.858 & 0 & 0.126 \\
\hline Wednesday & 0.837 & 0 & 0.142 \\
\hline Thursday & 0.943 & 0 & 0.050 \\
\hline Friday & 0.868 & 0 & 0.092 \\
\hline Saturday & 0.671 & 0 & 0.196 \\
\hline Sunday & 0.719 & 0 & 0.184 \\
\hline
\end{tabular}

$\hat{a}^{h}[t], \hat{b}^{h}[t]$, and $\hat{c}^{h}[t]$ denote the predicted parameters for the upcoming price tariff for each hour $h$ on day $t$. Our proposed price parameter prediction model is obtained as follows:

$\hat{a}^{h}[t]=k_{1} a^{h}[t-1]+k_{2} a^{h}[t-2]+k_{7} a^{h}[t-7], \quad \forall h \in \mathcal{H}$.

Here, $a^{h}[t-1], a^{h}[t-2]$, and $a^{h}[t-7]$ denote the previous values of parameter $a^{h}$ on yesterday, the day before yesterday, and the same day last week, respectively. In this regard, the expression in (17) formulates a weighted average price predictor filter with coefficients $k_{1}, k_{2}$, and $k_{7}$. Similar models can be obtained for predictions of parameters $\hat{b}^{h}[t]$ and $\hat{c}^{h}[t]$. However, in practice, the threshold parameter $c^{h}[t]$ is usually fixed and does not change on a daily basis. It may only change once or twice a year at different seasons.

Given $\hat{a}^{h}, \hat{b}^{h}$, and $\hat{c}^{h}$, the predicted price is derived as

$$
\hat{p}^{h}\left(l^{h}\right)= \begin{cases}\hat{a}^{h}, & \text { if } 0 \leq l^{h} \leq \hat{c}^{h}, \\ \hat{b}^{h}, & \text { if } l^{h}>\hat{c}^{h} .\end{cases}
$$

Here, the question is: Which choice of the filter coefficients leads to the best prediction? To answer this question, we applied the prices used by IPC for predicting parameter $a^{h}$ based on two different coefficient selection approaches. Recall that for the RTP tariff used by IPC, we have: $p^{h}\left(l^{h}\right)=a^{h}$. Hence, predicting parameter $a^{h}$ is enough to predict the prices.

1) All-days-same Coefficients: In this approach we assume that $k_{1}, k_{2}$, and $k_{7}$ are the same every day. By calculating the prediction error $\left|\hat{p}^{h}\left(l^{h}\right)-p^{h}\left(l^{h}\right)\right|$, we obtained the optimal coefficients in this case as $k_{1}=0.718, k_{2}=0, k_{7}=0.216$. The prediction error resulting from the expression in (17) when these coefficients are used observed to be $17 \%$ on average.

2) Each-day-different Coefficients: In this approach we select parameters $k_{1}, k_{2}$, and $k_{7}$ for each day of the week separately. The optimal choices of the prediction filter coefficients are shown in Table I. We can see that the coefficients are significantly different on Monday compared to the other days of the week. This is because unlike all the other days, there is a low correlation between the prices on Monday and the day before that due to lower prices on the weekends. For all other days, the prediction is only based on the prices on the last day and the prices on the same day last week. In fact, for the cases when the prices are highly correlated with those yesterday, there is really no need to know the price values the day before yesterday. Using the coefficients in Table I, we observed that the prediction error can reduce to only $13 \%$ on average. An example for predicting the prices on Tuesday

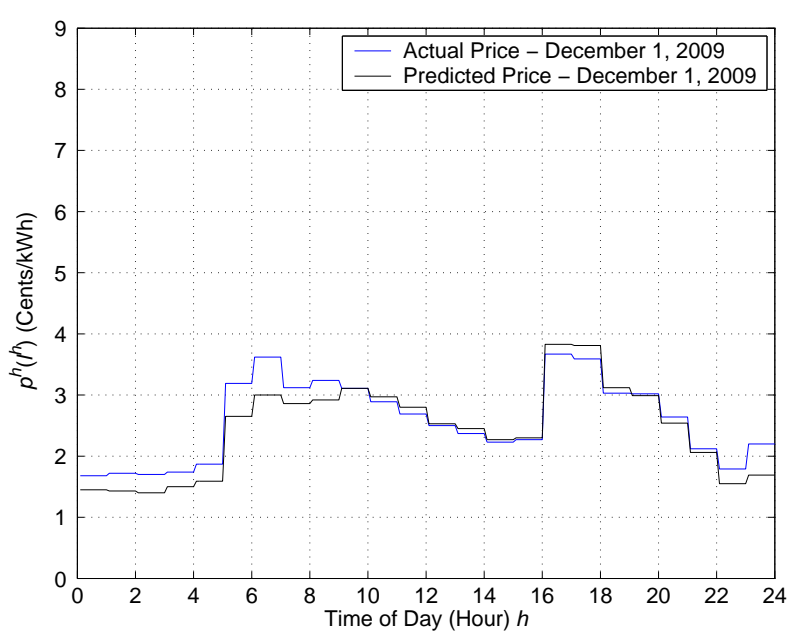

Fig. 6. Results when we use the weighted average price predictor filter in (17) with the choice of its coefficients according to Table I for predicting the real-time prices used by IPC on Tuesday December 1, 2009.

December 1, 2009 based on the price values within the last week of November 2009 are shown in Fig. 6.

From the above results we can conclude that the proposed weighted average price prediction filter structure in (17), with coefficients which are carefully selected based on the day of the week as in Table I, can provide an efficient predictor for residential load control. We also note that the proposed prediction structure has minimum computation complexity and its implementation only requires a lookup table for the daily coefficients, a limited memory, and the capability of performing simple arithmetic operations. Price prediction accuracy can further improve by using longer and more computationally complicated price prediction filters, if needed. Next, we will use the predicted prices in our proposed optimization-based residential energy consumption scheduling scheme.

\section{Optimal Residential LoAd CONTROL}

Recall from Section II-C that problem (15) is not tractable in its current form due to the non-differentiability of price function $p^{h}\left(l^{h}\right)$ in (6). In this section, we explain how we can solve problem (15) in practice. But first, we note that if the utility company does not release the prices for all upcoming hours within the scheduling horizon, i.e., if the price announcement horizon $P$ is strictly less than the scheduling horizon $H$, then part of objective function in (15) needs to be constructed based on the price prediction results which we discussed in Section III. In this regard, we can rewrite optimization problem (15) in general form as follows:

$$
\begin{aligned}
\underset{x \in \mathcal{X}}{\operatorname{minimize}} & \sum_{h=1}^{P} p^{h}\left(\sum_{a \in \mathcal{A}} x_{a}^{h}\right)\left(\sum_{a \in \mathcal{A}} x_{a}^{h}\right) \\
& +\sum_{h=P+1}^{H} \hat{p}^{h}\left(\sum_{a \in \mathcal{A}} x_{a}^{h}\right)\left(\sum_{a \in \mathcal{A}} x_{a}^{h}\right) \\
& +\lambda_{\text {wait }} \sum_{h=1}^{H} \sum_{a \in \mathcal{A}} \frac{\left(\delta_{a}\right)^{\beta_{a}-h} x_{a}^{h}}{E_{a}},
\end{aligned}
$$

where $p^{h}\left(l^{h}\right)$ and $\hat{p}^{h}\left(l^{h}\right)$ are as in (6) and (18), respectively. We notice that in the objective function in problem (19), 


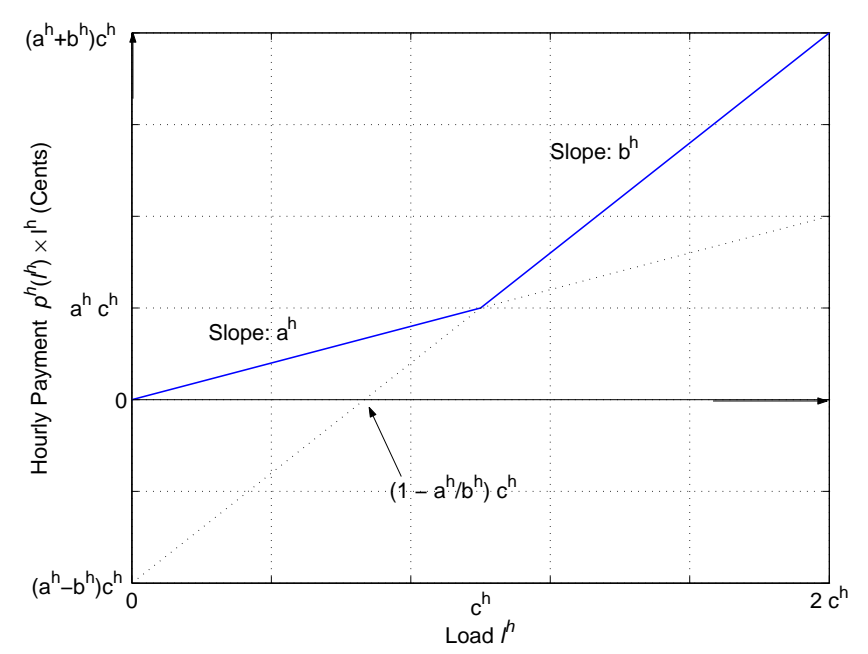

Fig. 7. An illustration of the hourly payment $p^{h}\left(l^{h}\right) \times l^{h}$ with inclining block rates. Notice that we always have $a^{h}>b^{h}$ for all conservation rates.

we have decomposed the total electricity payment within the scheduling horizon $\mathcal{H}$ into two parts. The first part shows the exact payment within the next $P$ hours while the second part shows the estimated payment within the $H-P$ hours after that. Clearly, same as that in problem (15), the objective function in optimization problem (19) is non-differentiable.

Next, consider the illustration of the hourly payment $p^{h}\left(l^{h}\right) \times l^{h}$ in Fig. 4, where $p^{h}\left(l^{h}\right)$ is as in (6). Note that in the IBR model, we always have $a^{h}<b^{h}$. Therefore, the hourly payment is formed based on two intersecting lines:

$$
\text { Payment }=a^{h} l^{h}
$$

and

$$
\text { Payment }=b^{h} l^{h}+\left(a^{h}-b^{h}\right) c^{h} .
$$

Therefore, for each $h \in \mathcal{P} \triangleq\{1, \ldots P\}$, we have

$$
p^{h}\left(l^{h}\right) \times l^{h}=\max \left\{a^{h} l^{h}, b^{h} l^{h}+\left(a^{h}-b^{h}\right) c^{h}\right\} .
$$

On the other hand, for each $h \in \mathcal{H} \backslash \mathcal{P}=\{P+1, \ldots, H\}$, we have

$$
\hat{p}^{h}\left(l^{h}\right) \times l^{h}=\max \left\{\hat{a}^{h} l^{h}, \hat{b}^{h} l^{h}+\left(\hat{a}^{h}-\hat{b}^{h}\right) \hat{c}^{h}\right\} .
$$

Therefore, problem (19) can be reformulated as

$$
\begin{aligned}
& \underset{x \in \mathcal{X}}{\operatorname{minimize}} \sum_{h=1}^{P} \max \left\{a^{h} \sum_{a \in \mathcal{A}} x_{a}^{h}, b^{h} \sum_{a \in \mathcal{A}} x_{a}^{h}+\left(a^{h}-b^{h}\right) c^{h}\right\} \\
&+ \sum_{h=P+1}^{H} \max \left\{\hat{a}^{h} \sum_{a \in \mathcal{A}} x_{a}^{h},\right. \\
&\left.\hat{b}^{h} \sum_{a \in \mathcal{A}} x_{a}^{h}+\left(\hat{a}^{h}-\hat{b}^{h}\right) \hat{c}^{h}\right\} \\
&+\lambda_{\text {wait }} \sum_{h=1}^{H} \sum_{a \in \mathcal{A}} \frac{\left(\delta_{a}\right)^{\beta_{a}-h} x_{a}^{h}}{E_{a}}
\end{aligned}
$$

Finally, by introducing auxiliary variables $v^{h}$ for all $h \in \mathcal{H}$, we can rewrite problem (24) as

$$
\begin{array}{rlrl}
\underset{\substack{x \in \mathcal{X} \\
v^{h}, \forall h \in \mathcal{H}}}{\operatorname{minimize}} & \sum_{h=1}^{H} v^{h}+\lambda_{\text {wait }} \sum_{h=1}^{H} \sum_{a \in \mathcal{A}} \frac{\left(\delta_{a}\right)^{\beta_{a}-h} x_{a}^{h}}{E_{a}} & \\
& a^{h} \sum_{a \in \mathcal{A}} x_{a}^{h} \leq v^{h}, & \forall h \in \mathcal{P}, \\
& b^{h} \sum_{a \in \mathcal{A}} x_{a}^{h}+\left(a^{h}-b^{h}\right) c^{h} \leq v^{h}, & \forall h \in \mathcal{P}, \\
& \hat{a}^{h} \sum_{a \in \mathcal{A}} x_{a}^{h} \leq v^{h}, & & \forall h \in \mathcal{H} \backslash \mathcal{P}, \\
& \hat{b}^{h} \sum_{a \in \mathcal{A}} x_{a}^{h}+\left(\hat{a}^{h}-\hat{b}^{h}\right) \hat{c}^{h} \leq v^{h}, & \forall h \in \mathcal{H} \backslash \mathcal{P} .
\end{array}
$$

We can prove by contradiction that problems (24) and (25) are equivalent [33, p. 130] and have exactly the same optimal solutions in terms of the scheduled energy consumptions. Interestingly, unlike problems (19) and (24), problem (25) is linear and differentiable. Therefore, it can be solved efficiently by using linear programming techniques [34]. In particular, the interior-point method [33, pp. 615-620] can be used to solve problem (25) in polynomial computation time.

\section{Remarks, Special Cases, and Extensions}

The proposed optimization-based residential load control framework in this paper can be extended in various directions. In this section, we overview a number of scenarios that can be addressed by slight modification in the system model.

\section{A. Appliances with Discrete Energy Consumption Levels}

Recall from Section II-A that in our system model, the hourly-based energy consumption scheduled for each appliance is a continuous variable which is lower-bounded by $\gamma_{a}^{\min }$ and is upper-bounded by $\gamma_{a}^{\max }$. This setting can easily be extended to the scenario where the scheduled energy consumption may only take the discrete values $\gamma_{a}^{\min }$ and $\gamma_{a}^{\max }$ when the appliance is "off" and "on", respectively. For each appliance $a \in \mathcal{A}$ and at each hour $h \in \mathcal{H}$, let $y_{a}^{h}$ denote an auxiliary binary variable such that $y_{a}^{h} \triangleq 1$ if appliance $a$ is "on" and $y_{a}^{h} \triangleq 0$ otherwise. By definition, the former requires an energy consumption level of $x_{a}^{h}=\gamma_{a}^{\min }$ while the latter requires an energy consumption level of $x_{a}^{h}=\gamma_{a}^{\max }$. Therefore, for each appliance $a \in \mathcal{A}$, the relationship between the energy consumption scheduling vector $\mathbf{x}_{a}$ and the auxiliary vector $\mathbf{y} \triangleq\left[y_{a}^{1}, \ldots, y_{a}^{H}\right]$ can be expressed as follows:

$$
x_{a}^{h}=y_{a}^{h} \gamma_{a}^{\max }+\left(1-y_{a}^{h}\right) \gamma_{a}^{\min }, \quad \forall h \in\left[\alpha_{a}, \beta_{a}\right] .
$$

From (26), if $y_{a}^{h}=1$ then $x_{a}^{h}=1 \times \gamma_{a}^{\max }+(1-1) \times \gamma_{a}^{\min }=$ $\gamma_{a}^{\max }$ and if $y_{a}^{h}=0$ then $x_{a}^{h}=0 \times \gamma_{a}^{\max }+(1-0) \times \gamma_{a}^{\min }=$ $\gamma_{a}^{\text {min }}$. By adding optimization variables $\mathbf{y}_{a} \triangleq\left[y_{a}^{1}, \ldots, x_{a}^{H}\right]$ as well as linear constraint (26) for each appliance $a \in \mathcal{A}$ to optimization problem (25), the case for discrete energy consumption levels can be fully incorporated in our formulation. In this case, the modified version of problem (25) would be a linear mixed integer program which is more complicated than 
a linear program, but still can be solved by using optimization software such as CPLEX [35] and MOSEK [36]. However, handling the computational complexity of these algorithms to be implemented in smart meters would remain a major challenge in case of using discrete energy consumption levels.

\section{B. Interruptible and Uninterruptible Residential Load}

Some load such as charging the battery for a PHEV are interruptible. That is, it is possible to charge the battery for one hour, then stop charging for another hour, and then finish the charging after that. However, if the load is uninterruptible, then as soon as the corresponding appliance starts operation, its operation needs to continue until it finishes. This requires imposing further limitations on the choices of the energy consumption scheduling vectors. Again, considering the case with discrete energy consumption levels as in Section, V-A, for each uninterruptible appliance $a$, let $\theta_{a}$ denote the duration of time, in number of hours, that appliance $a$ needs to operate at power level $\gamma_{a}^{\max }$. Also at each $h \in \mathcal{H}$, let $z_{a}^{h}$ denote an auxiliary binary variable such that $z_{a}^{h} \triangleq 1$ if appliance $a$ starts operation at hour $h$ and $z_{a}^{h} \triangleq 0$ otherwise. We have

$$
\sum_{h=\alpha_{a}}^{\beta_{a}-\theta_{a}+1} z_{a}^{h}=1,
$$

and

$$
z_{a}^{h}=0, \quad \forall h \in \mathcal{H} \backslash\left[\alpha_{a}, \beta_{a}-\theta_{a}+1\right] .
$$

That is, the operation of appliance $a$ may start some time between hours $\alpha_{a}$ and $\beta_{a}-\theta_{a}+1$ in order to make sure that it finishes operation by time $\beta_{a}$. We can relate the start time vector $\mathbf{z}_{a} \triangleq\left[z_{a}^{1}, \ldots, z_{a}^{H}\right]$ with auxiliary vector $\mathbf{y}_{a}$ as

$$
y_{a}^{h} \geq z_{a}^{h}, y_{a}^{h+1} \geq z_{a}^{h}, \ldots, y_{a}^{h+\theta_{a}-1} \geq z_{a}^{h}, \quad \forall h \in \mathcal{H} .
$$

From (29), if $z_{a}^{h}=1$, then $y_{a}^{h}=y_{a}^{h+1}=\ldots=y_{a}^{h+\theta_{a}-1}=1$. On the other hand, from (29) together with (26), we have $x_{a}^{h}=x_{a}^{h+1}=\ldots=x_{a}^{h+\theta_{a}-1}=\gamma_{a}^{\max }$. We conclude that although optimal energy scheduling for uninterruptible load requires adding some extra variables, the resulting optimization problem still remains a linear mixed-integer program.

\section{Availability of Multiple Retail Electricity Sources}

Consider the case where an end user has the ability to obtain electricity from a set of $\mathcal{S}$ utility companies simultaneously, where $|\mathcal{S}|>1$. We denote the total hourly load to each utility $s \in \mathcal{S}$ by $l_{s}^{h}$. Correspondingly, the real-time prices advertised by each utility $s$ is denoted by $p_{s}^{h}\left(l_{s}^{h}\right)$. Similarly, we define $x_{a, s}^{h}$ as the energy consumption scheduling variable corresponding to each appliance $a$ for energy consumption obtained from each utility $s$ at each time $h$. Clearly, if no IBR tariff is used by any of the utility companies, then it is an optimal choice for the end user to select $x_{a, s}^{h}=0$ for each $s \neq s_{h}^{\star}$ where

$$
s_{h}^{\star}=\min _{s \in \mathcal{S}} a_{s}^{h}, \quad \forall h \in \mathcal{H} .
$$

That is, it would be optimal for the end user to obtain all its energy need from the utility with lowest price. However, if IBR is adopted, then obtaining the optimal solution would be more complicated as it could be beneficial for the user to distribute its load among the available utility companies to avoid being charged with the rates at the higher block. Nevertheless, the reformulated version of optimization problem (25) for the case with multiple utility companies would still be a linear program.

\section{Avoiding Load Synchronization}

In general, it is desired for the power distribution and wiring systems such that no load synchronization occurs among different appliances in each household. As we will see in Section VI-D, adopting IBR model helps in avoiding the concentration of a large portion of energy consumption in a single low-price hour. On the other hand, it is also desired to even avoid synchronization among different appliances that start operation exactly at the same hour in order to prevent a sharp spike in the residential load. This can be done by introducing a short random starting delay, e.g., a few seconds, to diversify the starting moments among different appliances.

\section{E. Announcing the Scheduled Consumption Back to the Utility}

One of the main challenges that the utility companies face is the need for predicting the demand load by end users. Clearly, by knowing the upcoming demand, the utility companies and regional power plants can better perform energy dispatching. Such predictions may only be done statistically in the current electric grid. However, by large deployments of the automatic residential load control strategies that we proposed in this paper, the end users are potentially capable of announcing their upcoming load back to the utility company through a twoway digital communication infrastructure as in the one already shown in Fig. 2. More precisely, the end user can send its total upcoming daily load $\mathbf{l} \triangleq\left[l^{1}, \ldots, l^{H}\right]$ as a control message to the utility company. Given the expected load from all users, the utility company would have an accurate estimation of the load that it needs to provide within the next couple of hours. Therefore, the proposed load control structure can not only help users better respond to real-time prices, it can also potentially enable the utilities to have an idea on how the energy is and will be consumed by residential end users.

\section{F. Handling Load Reduction Requests}

Load reduction requests are usually sent out by the utilities when electricity demand is high enough to put the grid reliability at risk, or rising demand requires the imminent activation of expensive/unreliable generation sets. In a smart grid, an advance notice for load reduction can be sent through the communication infrastructure to each meter asking the energy scheduler to take an appropriate action. This can be done in our design by increasing the prices used in the optimization for the next two or three hours. This will automatically postpone a portion of the upcoming energy consumption to some later hours leading to a major decrease in the total load.

\section{G. Residential Electricity Storage}

As PHEVs become popular, there is an increasing interest in using the storage capacity of their batteries to return some 
energy back to the grid when needed [37]. In this setting, the users would buy electricity for charging their batteries at some low-price hours and then sell electricity back to the grid by discharging their batteries when the price is high. Therefore, the users can not only help balancing supply and demand in the regional electricity market, but also make money. However, due to the same reasons discussed in Section I, it is difficult for the users to keep monitoring the real-time prices in order to decide when it is the best time to charge or discharge their batteries. Our proposed optimization-based load control model can be extended in this regard by incorporating negative loads for discharging actions. Price prediction would still be helpful.

\section{H. Accommodating Changes in Users' Energy Needs}

The energy scheduler discussed in this paper can update schedules at any time based on the user's needs. For example, assume that an initial energy schedule is planned at 8:00 $\mathrm{AM}$ and the appliances are assigned to consume energy accordingly. Then, later at 11:00 AM the user decides to add scheduling the consumption for his dishwasher with $\alpha_{a}=$ 11:00 AM and $\beta_{a}=3: 00$ PM. In that case, the energy scheduler can adjust the existing choices of energy consumption schedules and solve problem (25) based on the new situation. Clearly, for those appliances that are already in the middle of their operation, parameter $E_{a}$ is recalculated accordingly.

\section{Simulation Results}

In this section, we present the simulation results and assess the performance of our proposed residential load control scheme with price prediction. Unless we state otherwise, the simulation setting is as follows. We consider a single household with various appliances and assume that it has subscribed for the RTP program adopted by IPC. We mainly focus on its consumption within a four months period from September 1, 2009 to December 31, 2009. In total, this duration includes 122 days. For the purpose of our study, we assume that the number of appliances used in this household at each day varies from 10 to 25 . They include certain appliances with fixed consumption schedules such as refrigerator-freezer (daily usage: $1.32 \mathrm{kWh}$ ), electric stove (daily usage: $1.89 \mathrm{kWh}$ for self-cleaning and $2.01 \mathrm{kWh}$ for regular), lighting (daily usage for 10 standard bulbs: $1.00 \mathrm{kWh}$ ), heating (daily usage: $7.1 \mathrm{kWh}$ ), etc. [29] as well as appliances with more flexible energy consumption scheduling requirements such as dishwasher (daily usage: 1.44 $\mathrm{kWh}$ ), clothes washer (daily usage: $1.49 \mathrm{kWh}$ for energy-star $1.94 \mathrm{kWh}$ for regular), clothes dryer (daily usage: $2.50 \mathrm{kWh}$ ), and PHEV (daily usage: $9.9 \mathrm{kWh}$ ), etc. [29] [2]. We assume that the scheduling horizon $H=24$. That is, the user solves optimization problem (25) to decide about his consumption for the next 24 hours. On the other hand, although the pricing model used by IPC is day-ahead (i.e., price announcement horizon $P=24$ ), we also address the cases where $P \ll 24$ which are better representations of the "real-time" pricing tariffs. Clearly, this would require price prediction as we already discussed in Section III. In addition, we further add IBR model to the real-time prices which increase by $40 \%$ at the higher block and the higher block starts at the hourly consumption of $2.5 \mathrm{kWh}$. Finally, in most cases, we assume that $\delta_{a}=1$ for all $a \in \mathcal{A}, \lambda_{\text {wait }}=1$, and $E^{\max } \gg 1$.

\section{A. Performance Gains from Users and Utility Prospectives}

We start by looking at the resulting payments from the users as well as the PAR in the residential load when we use our proposed load control model. Clearly, the user is interested in reducing its charges while the utility is interested in having a balanced load demand with a low PAR. The trends of daily electricity charges and PAR for a sample residential load based on the day-ahead real-time prices adopted by IPC from September 1 to December 31, 2009 are shown in Fig. 8. From the results in Fig. 8(a), by using the proposed load control scheme the user's average daily payment decreases by $25 \%$ from 108 cents to 81 cents. This is equivalent to reducing the monthly electricity payment from $\$ 32.4$ to only $\$ 24.3$. On the other hand, from the results in Fig. 8(b), the average PAR in daily load is reduced by $38 \%$ from 4.49 to 2.75 . Therefore, the user pays less and makes a more balanced load. Similar trends are observed in almost every day ${ }^{2}$. The results in this figure suggest that the deployment of the proposed residential energy consumption scheduling structure is beneficial for not only the end user but also for the utility company.

\section{B. Impact of Price Announcement Horizon \& Price Prediction}

For the experiment studied in Section VI-A, we assumed that the price announcement horizon $P=H=24$ hours. That is, we considered the day-ahead pricing model. In this section, we study the case when $P$ takes values smaller than the scheduling horizon $H$. As a result, it is required to use a price predictor, as in (17), in order to plan for the upcoming daily energy consumption. The filter coefficients can be selected daily according to Table I. Also recall that our price predictions showed an average prediction error of $13 \%$. Interestingly, when we use the predicted prices in load control, the optimality gap in the performance is significantly less. This is shown in Fig. 9 when we plotted the average monthly electricity payment versus the value of parameter $P$. As already shown in Fig. 8(a), if $P=H=24$, then the user's payment reduces by $25 \%$. As we decrease $P$, the residential load control would be partially based on predicted prices rather than actual prices. Therefore, due to prediction error, the resulted energy consumption scheduling solution may not be optimal. Nevertheless, we can see that even for the case when $P=2$, i.e., the utility company announces the real-time prices only for the next two hours, the increase in the user's monthly payment is only $1.3 \%$, i.e., less than 50 cents. On the other hand, we have also plotted the resulting monthly electricity payment when an automatic but static load control is being employed. In static load control, the pricing information used by the energy scheduler is not updated on an hourly or daily basis. Instead, some average seasonal off-line information (in

\footnotetext{
${ }^{2}$ The spike in the PAR on September 29th occurred because a major load was scheduled at 4:00 AM which happened to have a very low price. However, we can reduce PAR by increasing the price at the higher block in IBR. For example, if the price at the higher block is $100 \%$ (instead of $40 \%$ ) higher than that in the lower block, the PAR on September 29th reduces to only 2.3276 .
} 


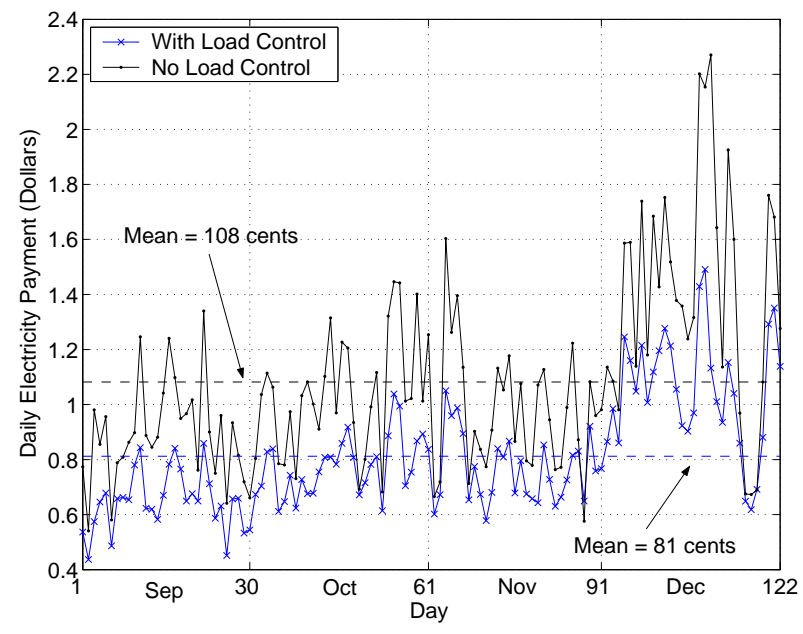

(a) Daily Electricity Charges.

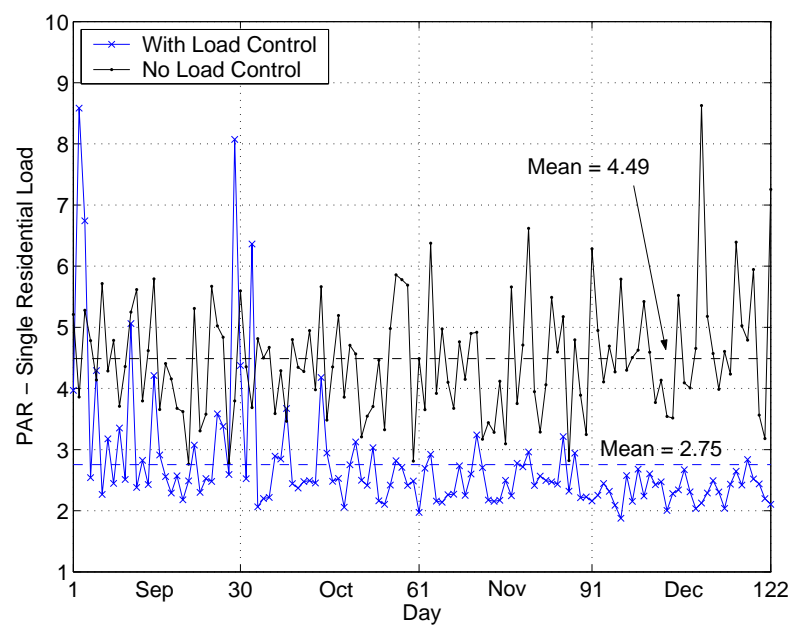

(b) Daily Peak-to-Average Ratio.

Fig. 8. Trends of daily charges and PAR for a sample residential load based on DAP adopted by IPC from September 1 to December 31, 2009.

our case, the average daily prices in the same season from last year) are being used for deciding on energy consumption schedules. From the results in Fig. 8, a static load control may reduce the household's energy cost only by $5.7 \%$. Therefore, it is indeed beneficial to facilitate on-line price updates, through a two-way digital communication infrastructure.

\section{The Impact of Scheduling Control Parameter $\delta_{a}$}

Recall from Section II-C that the user can balance payment and waiting time for the operation of each household appliance by adjusting parameter $\delta_{a}$ for each appliance $a$. By selecting $\delta_{a}=\delta=1$ for all $a \in \mathcal{A}$, the load control strategy only tries in reducing the electricity charges. As $\delta$ increases it will also be desired to finish the operation of each scheduled appliance sooner. Here, for the purpose of comparison, we define a waiting time (in percentage) for each appliance $a \in \mathcal{A}$ as

$$
\text { Waiting Time }=\frac{\mu_{a}-\alpha_{a}}{\beta_{a}-\alpha_{a}} \times 100,
$$

where $\mu_{a} \geq \alpha_{a}$ denotes the finishing time, i.e., the smallest hour $h$ such that $x_{a}^{h}=0$. Clearly, if the waiting time is $100 \%$,

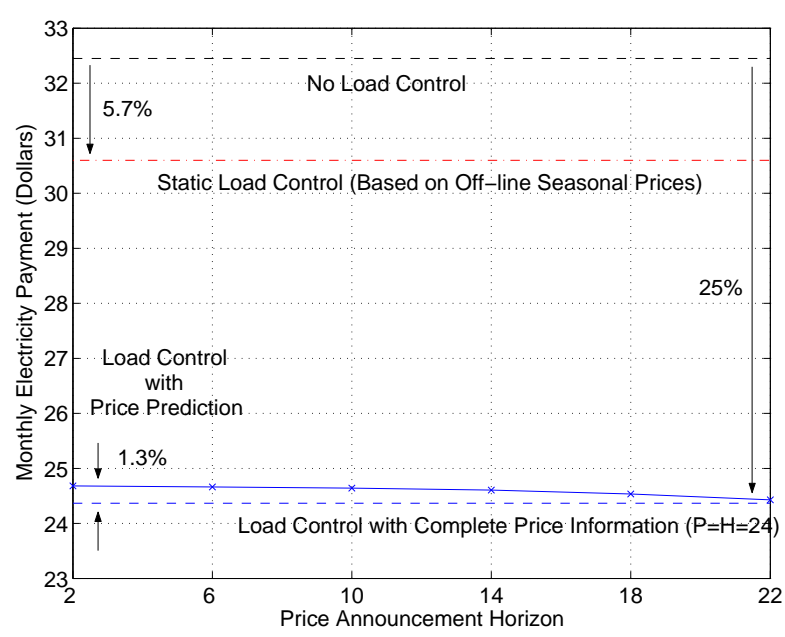

Fig. 9. The impact of changes in price announcement horizon $P<H$ in monthly electricity payments for different load approaches.

the scheduled energy consumption is such that the operation of the appliance $a$ finishes by the latest acceptable hour $\beta_{a}$. The results on monthly electricity payment and average waiting time are shown in Fig. 10. The trade-off is evident. As we increase $\delta$, the charges will increase while the waiting times decrease. For example, as we increase $\delta$ from 1 to 1.01 , the monthly payment increases from $\$ 24.36$ to $\$ 29.13$ while the waiting time decreases from $93.2 \%$ to only $17.5 \%$ of the valid scheduling interval. Of course, it is entirely up to the user to decide if he prefers paying less or instead getting the work done by the appliances within a shorter period of time.

\section{The Impact of Adopting Inclining Block Rates}

In this section, we show that combining RTP with IBR is indeed helpful in achieving more balanced residential load and avoiding load synchronization. This is shown in Fig. 11. We can see that an RTP tariff would lead to high PAR due to congestion at low-price hours. In fact, without IBR, the optimal solution of problem (25) is nothing but scheduling most of all energy consumption at hours with lower prices. This problem may not be visible in the existing manual residential load control programs as it is not easy or even possible for the users to keep watching the prices and only turn on their appliances when the prices are low [20], [21]. However, in an automatic load control such a congestion scenario can occur frequently and become troublesome. Therefore, it is significantly beneficial to combine RTP with IBR tariffs.

\section{E. The Impact of Number of Users}

So far, we have focused our simulation studies on scenarios with only a single user. In this section, we consider the case when a utility company serves 10 users which are all equipped with our proposed automatic residential load control capability. The simulation results are shown in Fig. 12. We can see from the PAR values in Fig. 12(a) that increasing the number of users can further balance the aggregated load even if no load control strategy is being deployed. This is due to the inherent randomness in different users' energy consumption 


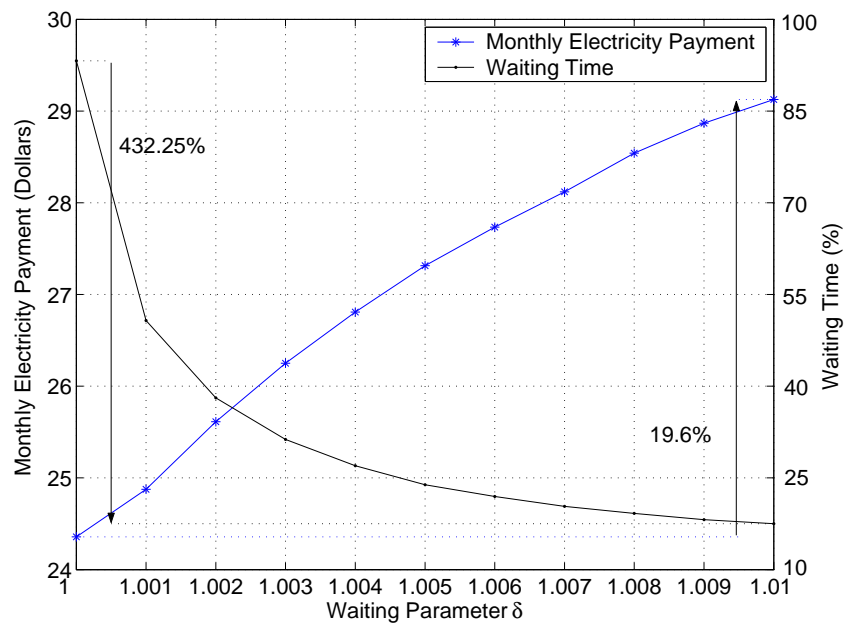

(a) Monthly Payment and Waiting Time versus Parameter $\delta$.

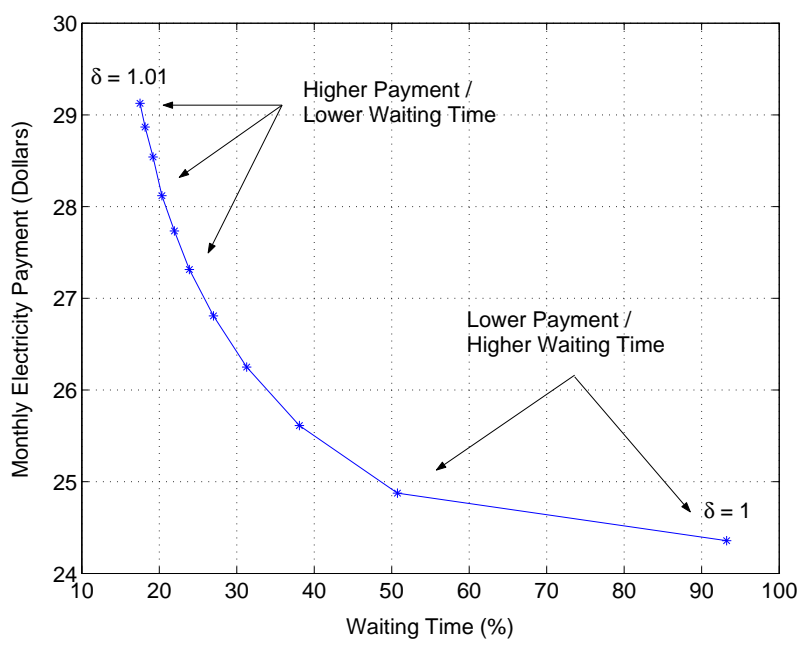

(b) Monthly Payment versus Waiting Time.

Fig. 10. The impact of changing control parameter $\delta$ on monthly electricity payment and waiting time. The trade-off is evident.

needs. Nevertheless, we can see that our proposed load control scheme can still be quite beneficial and reduce the PAR by $22 \%$. On the other hand, all users still pay less on their monthly electricity bills. Although, some users (mainly those who have more flexibility with respect to their energy consumption needs) benefit more in general.

\section{CONCLUSIONS}

Although real-time pricing has several potential advantages, its benefits are currently limited due to lack of efficient building automation systems as well as users' difficulty in manually responding to time-varying prices. Therefore, in this paper we proposed an optimal and automatic residential energy consumption scheduling framework which aims to achieve a trade-off between minimizing the payment and minimizing the waiting time for the operation of each household appliance based on the needs declared by users. We focused on a scenario when real-time pricing is combined with inclining block rates in order to have more balanced residential load with a low peak-to-average ratio. We argued that any load

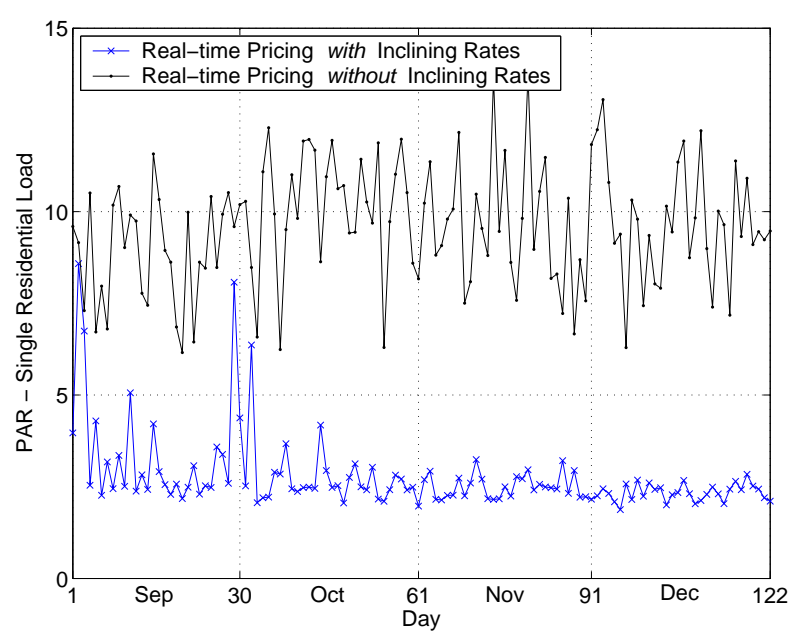

Fig. 11. The impact of adding IBR to RTP on achieved RAP.

control in real-time electricity pricing environments essentially requires some price prediction capabilities to enable planning ahead for the household energy consumption. By applying a simple and efficient weighted average price prediction filter to the actual hourly-based prices adopted by Illinois Power Company from January 2007 to December 2009, we obtain the optimal choices of the coefficients for each day of the week. Simulation results show that the combination of the proposed energy scheduler design and the price predictor leads to significant reduction in users' payments. This encourages the users to participate in the proposed residential load control program. Moreover, we observed that the peak-to-average ratio also decreases drastically which can provide the incentives for the utilities to support large-scale deployment of the designed energy schedulers in residential smart meters.

\section{REFERENCES}

[1] H. Allcott, "Real Time Pricing and Electricity Markets," Working Paper, Harvard University, Feb. 2009.

[2] A. Ipakchi and F. Albuyeh, "Grid of the future," IEEE Power and Energy Magazine, pp. 52-62, Mar. 2009.

[3] M. Fahrioglu and F. L. Alvardo, "Designing incentive compatible contracts for effective demand managements," IEEE Trans. on Power Systems, vol. 15, no. 4, pp. 1255-1260, Nov. 2000.

[4] B. Ramanathan and V. Vittal, "A framework for evaluation of advanced direct load control with minimum disruption," IEEE Trans. on Power Systems, vol. 23, no. 4, pp. 1681-1688, Nov. 2008.

[5] M. A. A. Pedrasa, T. D. Spooner, and I. F. MaxGill, "Scheduling of demand side resources using binary particke swarm optimization," IEEE Trans. on Power Systems, vol. 24, no. 3, pp. 1173-1181, Aug. 2009.

[6] H. Houthakker, "Electricity tariffs in theory and practice," The Economic Journal, vol. 61, no. 241, pp. 1-25, 1951.

[7] P. Steiner, "Peak loads and efficient pricing," Quarterly Journal of Economics, vol. 71, no. 4, pp. 585-610, 1957.

[8] S. Borenstein, "The long-run effects of real-time electricity pricing," Center for the Study of Energy Markets - Working Paper 133, 2004.

[9] F. Wolak, "Residential customer response to real-time pricing: The anaheim critical peak pricing experiment," Center for the Study of Energy Markets - Working Paper 151, May 2006.

[10] W. Burke and D. Auslander, "Residential electricity auction with uniform pricing and cost constraints," in Proc. of North American Power Symposium, Starkville, MS, Oct. 2009.

[11] P. Centolella, "The integration of price responsive demand into regional transmission organization (RTO) wholesale power markets and system operations (article in press)," Energy, 2009. 


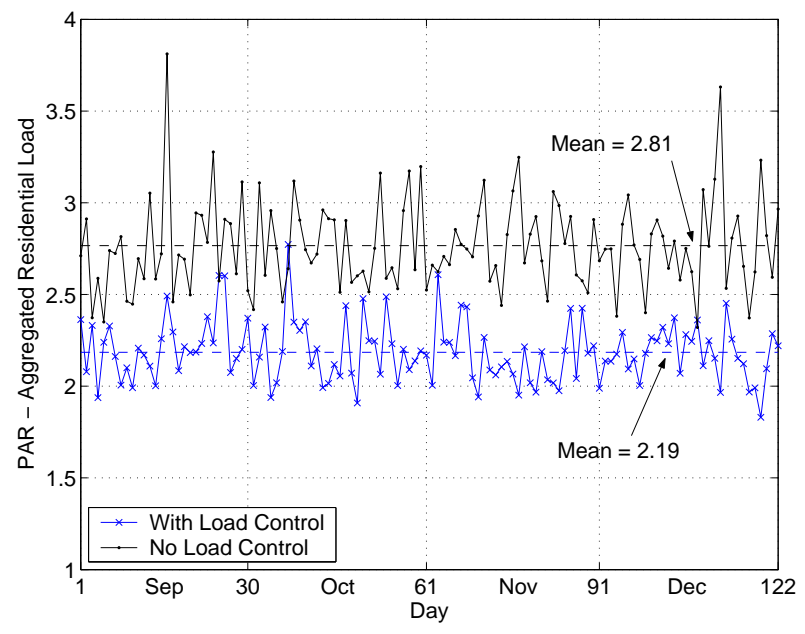

(a) Peak-to-average Ratio in Aggregated Load Demand.

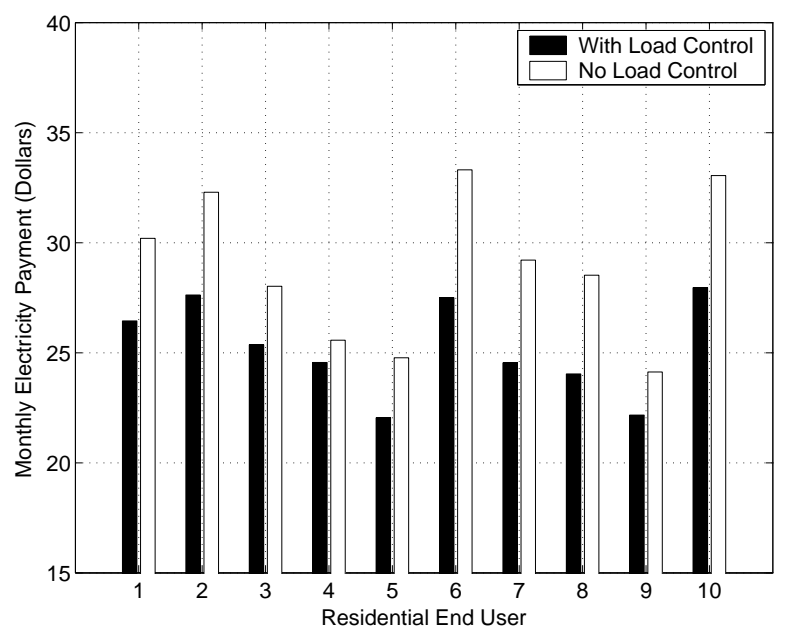

(b) Monthly Electricity Payments.

Fig. 12. Simulation results when the number of users increases to 10 .

[12] B. Alexander, "Smart meters, real time pricing, and demand response programs: Implications for low income electric customers," Oak Ridge National Laboratory Technical Report, Feb. 2007.

[13] S. Holland and E. Mansur, "Is real-time pricing green? the envirobmental impacts of electricity demand variance," Review of Economics and Statistics, vol. 90, no. 3, pp. 550-561, Aug. 2008.

[14] "Real-time pricing for residential customers, ameren illinois power co." http://www.ameren.com/Residential/ADC_RTP_Res.asp, Jan. 2010.

[15] "Smarter Electricity Pricing Coming to Ontario: McGuinty Government Rolls Out Time-of-Use Rates," Ontario Newsroom For Residents, 2009.

[16] P. Reiss and M.White, "Household electricity demand, revisited," Review of Economic Studies, vol. 72, no. 3, pp. 853-883, July 2005.

[17] A. H. Mohsenian-Rad, V. Wong, J. Jatskevich, and R. Schober, "Optimal and autonomous incentive-based energy consumption scheduling algorithm for smart grid," in Proc. of IEEE PES Conference on Innovative Smart Grid Technologies, Gaithersburg, MD, Jan. 2010.

[18] S. Borenstein, "Equity effects of increasing-block electricity pricing," Center for the Study of Energy Markets - Working Paper 180, Nov. 2008.

[19] BC Hydro, Electricity Rates, 2009.

[20] "Demand response program evaluationfinal report," Quantum Consulting Inc. and Summit Blue Consulting, LLC Working Group 2 Measurement and Evaluation Committee and California Edison Company, Apr. 2005.

[21] M. Ann-Piette, G. Ghatikar, S. Kiliccote, D. Watson, E. Koch, and D. Hennage, "Design and operation of an open, interoperable automated demand response infrastructure for commercial buildings," Journal of Computing \& Information Science in Eng., vol. 9, pp. 1-9, June 2009.
[22] M. Toney, "Panel presentation at the IEEE PES" conference on innovative smart grid technologies," Gaithersburg, MD, Jan.

[23] R. Krishnan, "Meters of Tomorrow," IEEE Power and Energy Magazine, pp. 92-94, Mar. 2008.

[24] U.S. Department of Energy, The Smart Grid: An Introduction, 2009.

[25] A. Vojdani, "Smart integration," IEEE Power and Energy Magazine, pp. 72-79, Nov. 2008.

[26] L. H. Tsoukalas and R. Gao, "From smart grids to an energy internet: Assumptions, architecrures, and requirements," in Third International Conference on Electric Utility Deregulation and Restructuring and Power Technologies, Apr. 2008.

[27] https://www2.ameren.com/RetailEnergy/realtimeprices.aspx, Jan. 2010.

[28] S. Tiptipakorn and W. J. Lee, "A residential consumer-centered load control strategy in real-time electricity pricing environment," in Proc. of North American Power Symposium, Las Cruces, NM, Oct. 2007.

[29] Office of Energy Efficiency, Natural Resources Canada, Energy Consumption of Major Household Appliances Shipped in Canada, 2005.

[30] ZigBee Alliance, "Zigbee smart energy profile specification, revision 15, zigbee document 075356r15," Dec. 2008.

[31] ZigBee Plus HomePlug Joint Working Group, "Smart energy profile marketing requirements document, draft revision 1.0," Mar. 2009.

[32] G. A. McNaughton and R. Saint, "Enterprise integration implications for home-area network technologies," in Proc. of IEEE PES Conference on Innovative Smart Grid Technologies, Gaithersburg, MD, Jan. 2010.

[33] S. Boyd and L. Vandenbergher, Convex Optimization. Cambridge University Press, 2004.

[34] D. Bertsimas and J. N. Tsitsiklis, Introduction to Linear Optimization. Belmont, MA: Athena Science, 1997.

[35] "ILOG CPLEX." http://www.ilog.com/products/cplex/, 2009.

[36] "MOSEK." http://www.mosek.com, 2009.

[37] S. Han, S. H. Han, and K. Sezaki, "Design of an optimal aggregator for vehicle-to-grid regulation service," in Proc. of IEEE PES Conference on Innovative Smart Grid Technologies, Gaithersburg, MD, Jan. 2010.

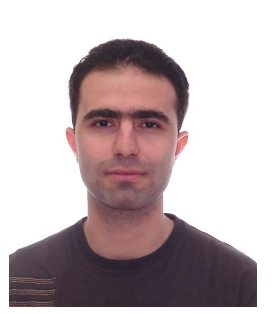

Amir-Hamed Mohsenian-Rad (S04-M09) received masters degree in Electrical Engineering from Sharif University of Technology in 2004 and Ph.D. degree in Electrical and Computer Engineering from The University of British Columbia (UBC) in 2008. From March to July 2007, he was a visiting student at Princeton University. Currently, Dr. Mohsenian$\mathrm{Rad}$ is a post-doctoral fellow at the University of Toronto. He is the recipient of the UBC Graduate Fellowship, Pacific Century Graduate Scholarship, and the Natural Sciences and Engineering Research Council of Canada (NSERC) fellowship. His research interests include design and optimization of communication networks and smart power systems.

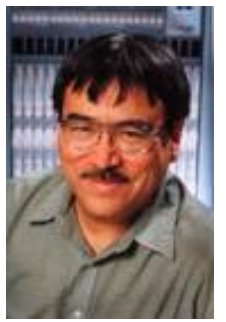

Alberto Leon-Garcia (F99) is a Professor in Electrical and Computer Engineering at the University of Toronto. He is a Fellow of the IEEE "For contributions to multiplexing and switching of integrated services traffic". He is also a Fellow of the Engineering Institute of Canada. He has received the 2006 Thomas Eadie Medal from the Royal Society of Canada and the 2010 IEEE Canada A. G. L. McNaughton Gold Medal for his contributions to the area of communications. He holds a Canada Research Chair in Autonomic Service Architecture. From 1999 to 2002, he was founder and CTO of AcceLight Networks in Ottawa which developed an all-optical fabric multi-terabit, multiservice core switch. He holds several patents and has published research extensively in the areas of switch architecture and traffic management. Professor Leon-Garcia's current research interests are focused on application-oriented networking and autonomic resources management with a focus on enabling pervasive smart infrastructure. His research team is currently developing a network testbed that will enable at-scale experimentation in new network protocols and distributed applications. Professor Leon-Garcia is recognized as an innovator in networking education. In 1986, he led the development of the University of Toronto - Northern Telecom Network Engineering Program. He has also led in 1997 the development of the Master of Engineering in Telecommunications program, and the communications and networking options in the undergraduate computer engineering program. $\mathrm{He}$ is the author of the leading textbooks: Probability and Random Processes for Electrical Engineering, and Communication Networks: Fundamental Concepts and Key Architecture. 